\title{
ARTíCULO \\ Distribución de tallas y potencial reproductivo de hembras de langosta verde Panulirus gracilis (Decapoda: Palinuridae) en Santa Rosa, Manabí, Ecuador
}

Size distribution and reproductive potential of green lobster Panulirus gracilis (Decapoda: Palinuridae) female in Santa Rosa, Manabí, Ecuador

\section{David Mero-del Valle ${ }^{1}$, Ricardo Castillo-Ruperti ${ }^{1}$ y Juan Figueroa-Pico ${ }^{1}$}

\begin{abstract}
${ }^{1}$ Departamento Central de Investigación, Universidad Laica Eloy Alfaro de Manabí, Ciudadela Universitaria Vía San Mateo, Manta, Ecuador, 130804 EC. david.mero@uleam.edu.ec

Abstract.- Spiny lobster Panulirus gracilis is a fishing resource of high commercial importance in Ecuador. Studies of fecundity and reproductive potential of population are fundamental and ensures sustainable use for the fishery, because it is intimately related with the renewal capacity of population. We analyzed size distribution and reproductive potential of spiny lobsters P. gracilis catched by nets in Santa Rosa, Manabí, Ecuador. Data was collected during July 2011, August, September and November 2012, and a sample of 273 females was obtained. Size of lobsters varied between 58 and $102 \mathrm{~mm}$ of carapace length (CL), with an average of $83 \mathrm{~mm} \mathrm{CL} \pm 7.1$; only $2 \%$ of lobsters had legal size (> $103 \mathrm{~mm} \mathrm{LC}$ ). Thirty percent of females were ovigerous stage III. Fecundity varied between 123,255 and 805,632 eggs, with an average of 397,115 \pm 148,069 eggs; relative fecundity was $1193 \pm 236$ eggs for each gram of weight. Reproductive potential analysis showed that females with size $<80 \mathrm{~mm} \mathrm{CL}$ contribute only with $17.8 \%$ of total egg production while females between 90 and $100 \mathrm{~mm} \mathrm{CL}$ provide $38.2 \%$ of total eggs production.
\end{abstract}

Key word: Reproductive potential, fecundity, Panulirus gracilis, Ecuador

\begin{abstract}
Resumen.- La langosta espinosa Panulirus gracilis es un recurso pesquero de gran importancia comercial en Ecuador. Estudios de fecundidad y el potencial reproductivo son fundamentales y garantiza su aprovechamiento sustentable para la pesquería, ya que está relacionado con la capacidad de renovación de la población. En el presente trabajo se analizó la distribución de tallas y el potencial reproductivo de P. gracilis capturadas en Santa Rosa, Manabí, Ecuador. La toma de datos se realizó durante julio 2011, agosto, septiembre y noviembre 2012, se obtuvo una muestra de 273 hembras. La talla varió entre 58 y $102 \mathrm{~mm}$ de longitud cefalotorácica (LC) con un valor promedio de $83 \mathrm{~mm} \mathrm{LC} \pm 7,1$ y sólo el $2 \%$ de la captura se encontró dentro de la talla legal permitida (> $103 \mathrm{~mm} \mathrm{LC}$ ). El 30\% de las hembras se encontraron en estadio reproductivo III, con una fecundidad absoluta entre 123.255 y 805.632 huevos y un promedio de $397.115 \pm 148.069$ huevos, la fecundidad relativa promedio fue de $1193 \pm 236$ huevos por cada gramo de peso del animal. El análisis del potencial reproductivo indica que las hembras menores de $80 \mathrm{~mm} \mathrm{LC}$ aportan sólo el $17,8 \%$ de la producción total de huevos, mientras que las hembras entre 90 y $100 \mathrm{~mm} \mathrm{LC}$ aportan con el $38,2 \%$.
\end{abstract}

Palabras clave: Potencial reproductivo, fecundidad, Panulirus gracilis, Ecuador

\section{INTRODUCCIÓN}

La langosta verde Panulirus gracilis (Streets, 1871) y Panulirus penicillatus (Olivier, 1791) son recursos pesqueros de gran importancia económica en Ecuador continental e insular, siendo esta última especie exclusiva de Galápagos. Según las estadísticas del Banco Central del Ecuador la exportaciones de $P$. gracilis y $P$. penicillatus entre el 2000 a 2009 ascienden a 985 toneladas (Ramírez et al. 2012).
En la costa continental del Ecuador la captura de $P$. gracilis es realizada por la flota pesquera artesanal, que opera dentro de las 8 millas náuticas utilizando redes de polifilamento de fondo y buceo semiautónomo (compresor tipo hookah) (Correa et al. 1994). No obstante, la tecnificación de la flota artesanal con el uso de buceo semiautónomo tuvo como consecuencia el aumento del esfuerzo pesquero en toda la costa continental ecuatoriana, teniendo como resultado la sobreexplotación del recurso, obligando a las poblaciones de $P$. gracilis a 
reproducirse en tallas cada vez más pequeñas como respuesta a la alta presión pesquera (Figueroa \& Mero 2013). Actualmente, el recurso langosta en la costa continental del Ecuador carece de efectivas políticas de vigilancia y manejo sustentable (Castillo et al. 2013). Seijo et al. (1997) y Cadima (2003) señalan que es necesario articular e integrar factores biológicos y ecológicos con aspectos socioeconómicos e institucionales, para disponer de información básica de la especie con el propósito de lograr establecer un plan de manejo sustentable de este recurso pesquero.

Las investigaciones realizadas para $P$. gracilis incluyen tallas de captura y artes de pesca (Figueroa \& Mero 2013), índices de actividad reproductiva (Naranjo 2012), estructura del hábitat y biología pesquera (Cun \& Campos 1993, Villón et al. 2000, Guzmán et al. 2008, Castillo et al. 2013), estudio de cadena de valor de la pesquería (Castrejón 2012), crecimiento y aspectos socioeconómicos (Briones \& Lozano 2003, Figueroa et al. 2013). Sin embargo, aún no se ha determinado el potencial reproductivo y la fecundidad de la especie, aspecto fundamental de la reproducción de recursos marinos que se encuentran sometidos a presión pesquera, porque están directamente relacionados con la capacidad de renovación de la población y sostenibilidad de la pesquería (Gracia 1985).

La fecundidad y en consecuencia el potencial reproductivo está condicionado a diversos factores intrínsecos de cada especie, tales como la talla, el peso, la edad e inclusive la latitud en que se encuentra su distribución (De Martini et al. 2003). Por tales motivos, el objetivo del presente estudio fue determinar el potencial reproductivo y la estructura de tallas de $P$. gracilis capturadas en Santa Rosa, Manabí, con la finalidad de contribuir con información biológica esencial para definir estrategias para la conservación y manejo sustentable del recurso.

\section{Materiales Y MÉTODOS}

\section{ÁREA DE ESTUDIO Y ASPECTOS GENERALES DE LA PESQUERÍA}

Durante julio 2011, agosto, septiembre y noviembre 2012 se monitorearon las capturas con redes de Panulirus gracilis provenientes de Santa Rosa, Manabí, Ecuador $\left(1^{\circ} 07^{\prime} 38,93 " \mathrm{~S}, 80^{\circ} 53^{\prime} 57,22^{\prime \prime W}\right)$ (Fig. 1). La captura comercial de $P$. gracilis en esta caleta pesquera se ha realizado desde 1960 con el uso de redes de fondo como arte de pesca tradicional, sin embargo, a partir de 1980 con la tecnificación de la flota pesquera artesanal se implementó la captura con equipo de buceo semiautónomo (compresor tipo hookah) (Cun \& Campos 1993).

Desde noviembre 2001 entró en vigencia el Acuerdo Ministerial N ${ }^{\circ} 182$ (Ministerio de Comercio Exterior Industrialización y Pesca 2001) $)^{1}$. en el cual se establece un periodo de veda total desde el 16 de enero hasta el 16 de junio de cada año, donde se prohíbe la tenencia, procesamiento, transporte y comercialización interna y externa. Además, transcurrido el periodo de veda solo se permitirá la tenencia, procesamiento, transporte y comercialización interna y externa de animales que tengan una talla mayor a $26 \mathrm{~cm} \mathrm{LT} \mathrm{o} 15 \mathrm{~cm}$ de cola y hembras no ovígeras.

La constante demanda de este recurso y el aumento del esfuerzo de pesca (número de pescadores, embarcaciones, días de pesca) ha ocasionado que la presión pesquera sobre el recurso esté provocando desequilibrios biológicos en las poblaciones de $P$. gracilis tales como disminución en la talla media de captura y de la talla media de madurez sexual y una alta frecuencia de especímenes por debajo de la talla legal (Figueroa \& Mero 2013).

\section{Procedimiento de muestreo}

Se registró la longitud cefalotorácica (LC) de los organismos desde la escotadura entre los cuernos surpraorbitales hasta el extremo posterior del cefalotórax. Los datos fueron tomados con un calibrador vernier (precisión $\pm 0,01 \mathrm{~mm}$ ). Los valores se expresaron en milímetros (mm). El sexo de los organismos se determinó mediante características macroscópicas externas (Figueroa et al. 2013). Para evaluar la actividad reproductiva, en las hembras se determinó el estado de madurez sexual según la escala propuesta por Briones et al. (1981): 1) hembras ovígeras (estadios III, IV, V); 2) hembras no ovígeras (estadios I, II, VI). La fecundidad se determinó analizando 82 hembras en estadio reproductivo III siguiendo los procedimientos de Domínguez (2003): se extrajo la masa de huevos de cada animal, se desprendieron los huevos de las setas de los pleópodos y las masas de huevos fueron preservadas en alcohol al 70\%, posteriormente las muestras se tamizaron y deshidrataron en una estufa a

${ }^{1}$ Ministerio de Comercio Exterior Industrialización y Pesca. 2001. Acuerdo Ministerial N 182 ., Subsecretaría de Recursos Pesqueros, Quito. <http://simce.ambiente.gob.ec/sites/default/files/documentos/anny/acuerdo-ministerial\%20182-vedade-la-langosta.pdf> 


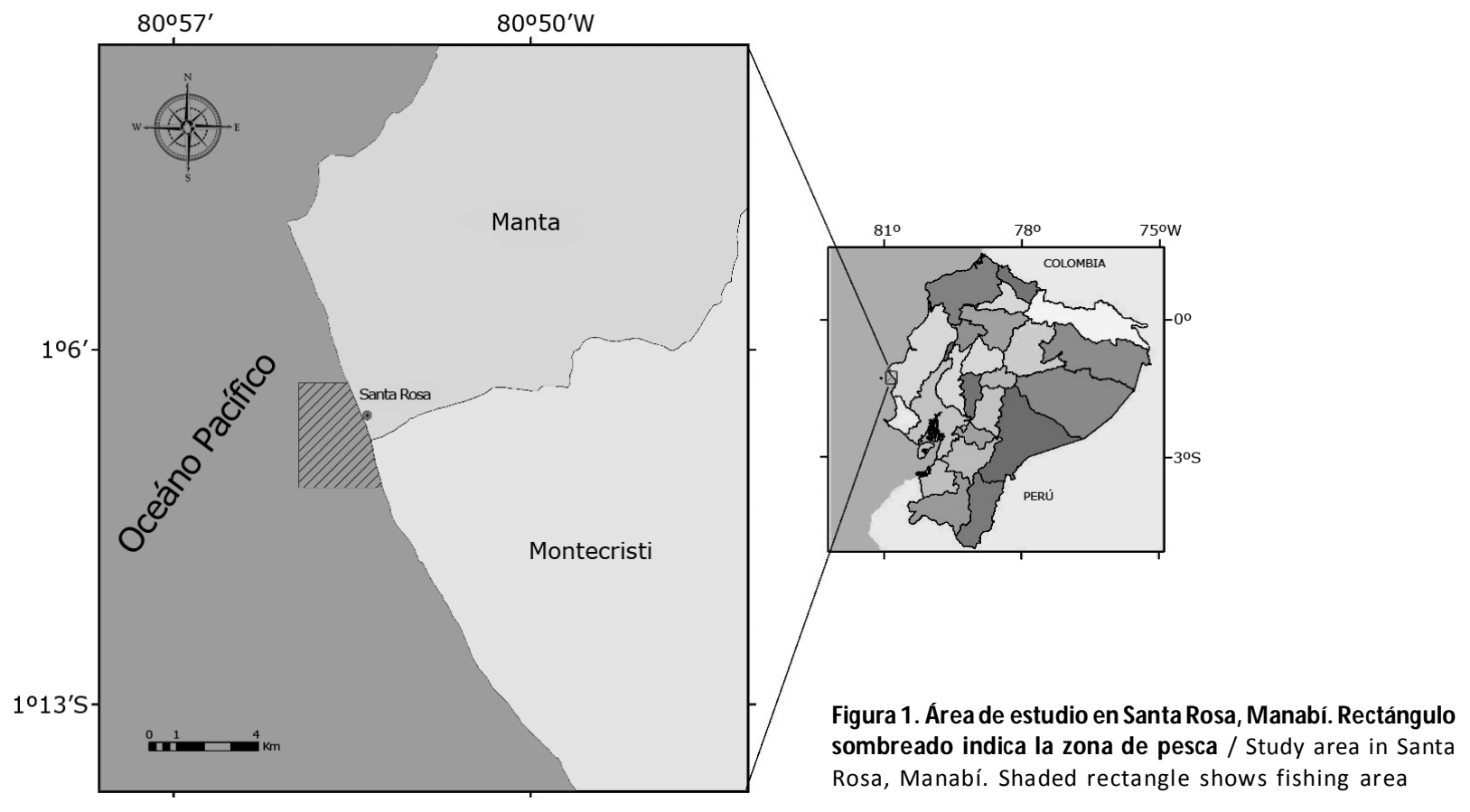

$45^{\circ} \mathrm{C}$. La masa de huevos se removió 2 veces por día para que la deshidratación fuese uniforme y lograr un peso constante de toda la muestra, todo el proceso se realizó cuidadosamente con el fin de perder la menor cantidad de huevos.

Posteriormente se pesaron 3 submuestras de $0,01 \mathrm{~g}$ de cada masa de huevos y se realizó el conteo manual de huevos presentes en cada submuestra utilizando microscopio estereoscopio MULTEC (modelo: ME7C-W). Se obtuvo el promedio del número de huevos de cada submuestra y este valor se extrapoló al peso total de la masa de huevos para obtener una estimación del número total de huevos de cada hembra.

\section{Análisis de eSTAdísticos}

La estructura de tallas y estadios reproductivos se analizaron con histogramas de distribución de frecuencias.

La fecundidad absoluta se determinó con la ecuación propuesta por De Martini et al. (1993):

$$
\mathrm{F}_{\mathrm{A}}=\mathrm{F}_{\mathrm{s}}\left(\frac{\mathrm{W}_{\mathrm{t}}}{\mathrm{W}_{\mathrm{s}}}\right)
$$

donde:

$\mathrm{F}_{\mathrm{s}}=$ número promedio de huevos de cada submuestra, $\mathrm{W}_{\mathrm{t}}=$ peso total de la masa de huevos $\mathrm{y} \mathrm{W}_{\mathrm{s}}=$ peso de los huevos de cada submuestra.

Se determinó la fecundidad relativa como el número de huevos producidos por cada gramo de peso de la hembra. Además, se estimó la relación entre la fecundidad absoluta y la longitud cefalotorácica usando un modelo lineal para obtener un mejor ajuste (Green et al. 2009).

El aporte de huevos a la población por clase de talla de hembras se determinó mediante el Índice de Potencial Reproductivo (IPR) propuesto Kanciruk \& Herrnkind (1976):

$$
\mathrm{IPR}=(\text { A.B.C }) / \mathrm{D}
$$

donde $\mathrm{A}=$ número de hembras por clase de talla/total de hembras, $\mathrm{B}=$ proporción de hembras ovígeras por clase de tallas, $\mathrm{C}=$ promedio de número de huevos por clase de talla y $\mathrm{D}=$ constante estandarizada.

El valor de la constante $\mathrm{D}=1511,6$ se obtuvo de la clase de talla de $80-85 \mathrm{~mm}$ LC debido a que presenta la mayor frecuencia de captura, la cual se estandarizó como el $100 \%$ para calcular el IPR, también se estimó el 
porcentaje de la producción total de huevos por clase de tallas (E) y el índice de productividad (F) que es la división del porcentaje de producción por clase de talla (E) entre la proporción del número de hembras por clase de talla (A) (Kanciruk \& Herrnkind 1976).

Esta ecuación asume un desove por año, sin embargo P. gracilis presenta 3 y 4 desoves al año $(<70 \mathrm{~mm} \mathrm{LC} \mathrm{y}\rangle$ $70 \mathrm{~mm}$ LC, respectivamente) (García 2004), motivo por el cual, el valor de IPR se calculó considerando este número de desoves.

\section{Resultados}

Se registró la información de 273 hembras, la talla varió entre de 58 y $102 \mathrm{~mm} \mathrm{LC}$, con un promedio de $83 \mathrm{~mm} \mathrm{LC} \pm$ 7,1 . La mayor frecuencia de captura se encontró en el intervalo de $70-85 \mathrm{~mm}$ LC con el $63 \%$ y sólo el $2 \%$ de la captura en Santa Rosa se encontró dentro de la talla legal permitida (> $103 \mathrm{~mm}$ LC equivalente a $260 \mathrm{~mm}$ de longitud total) (Fig. 2).

De las 273 hembras estudiadas, 113 fueron ovígeras, de las cuales el $28 \%$ presentó una talla menor a $80 \mathrm{~mm} \mathrm{LC}$. El porcentaje de hembras en estadio reproductivo III fue del 30\%; los estadios IV y V presentaron el 5 y $6 \%$, respectivamente (Fig. 3).

La talla mínima y máxima de las hembras en estadio III utilizadas para determinar la fecundidad estuvo entre 65,9 y 98,4 mm LC, con un promedio de 84,2 $\mathrm{mm} \mathrm{LC} \pm 7,4$.

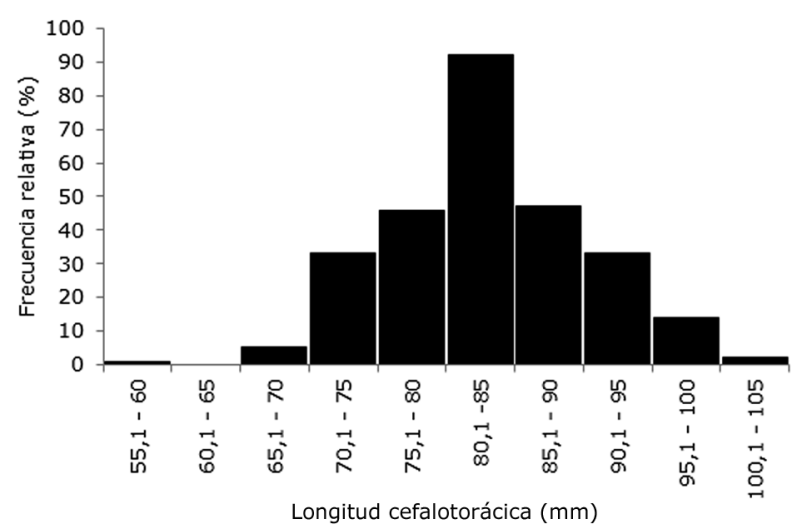

Figura 2. Distribución de tallas de hembras de Panulirus gracilis durante julio 2011, agosto, septiembre y noviembre 2012 capturadas en Santa Rosa, Manabí / Size distribution of female of Panulirus gracilis during July 2011, August and September 2012 captured in Santa Rosa, Manabí
La fecundidad absoluta fluctuó entre 123.255 y 805.632 huevos, la media fue de $397.116 \pm 148.069$ huevos. Las hembras entre 80 y $85 \mathrm{~mm}$ LC presentaron una fecundidad promedio de $382.095 \pm 74.026$. El análisis de regresión lineal entre la fecundidad y LC indican una correlación entre variables de $82 \%$ y el coeficiente de determinación es del $67 \%$ (Fig. 4). La producción mínima de huevos coincidió con la hembra de menor tamaño (65,9 mm LC), sin embargo, la hembra de mayor tamaño $(98,4 \mathrm{~mm} \mathrm{LC})$ no presentó la máxima producción de huevos (Fig. 4). La fecundidad relativa se estimó en 1193 huevos $\mathrm{g}^{-1}( \pm 236$ huevos $\mathrm{g}^{-1}$ ).

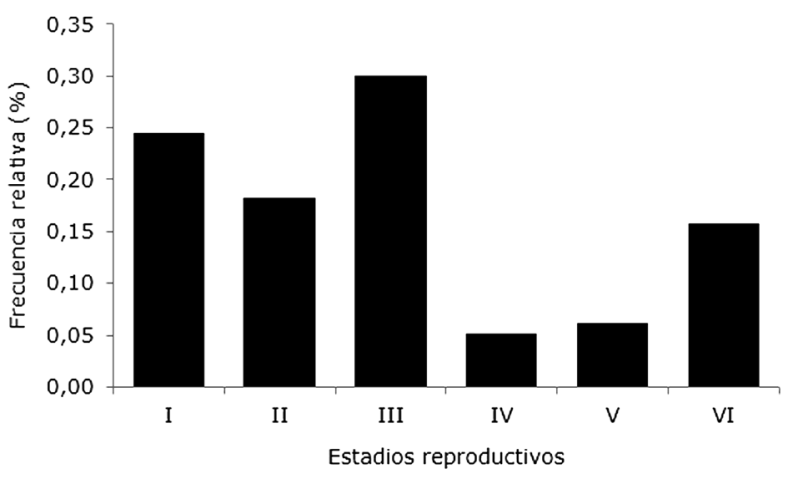

Figura 3. Distribución de frecuencias para los estadios reproductivos de hembras de Panulirus gracilis en Santa Rosa, Manabí / Reproductive stages distribution for female lobsters Panulirus gracilis in Santa Rosa, Manabí

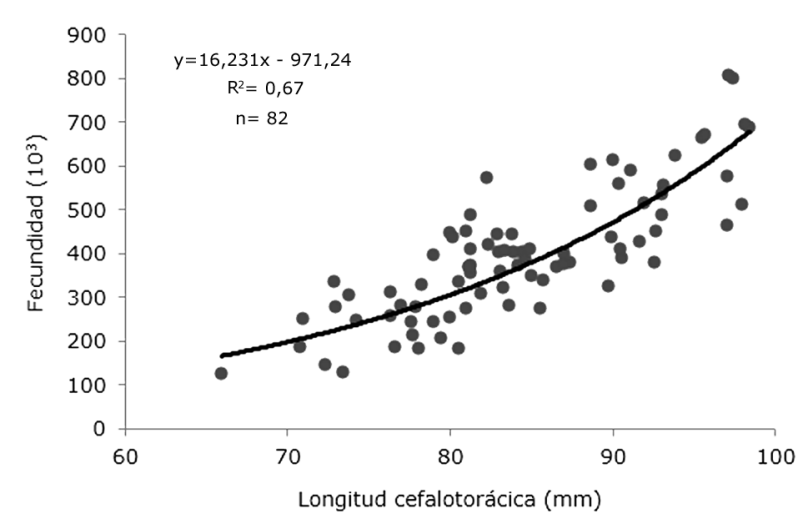

Figura 4. Relación entre la fecundidad (número de huevos) y la longitud cefalotorácica de la langosta verde Panulirus gracilis en Santa Rosa, Manabí / Relationship between fecundity (number of eggs) and carapace length of female spiny lobster Panulirus gracilis in Santa Rosa, Manabí 
Tabla 1. Estimación del índice de potencial reproductivo (IPR) para Panulirus gracilis en Santa Rosa, Manabí. A=número de hembras por clase de talla/total de hembras, $\mathrm{B}=$ proporción de hembras ovígeras por clase de tallas, $\mathrm{C}=$ promedio de número de huevos por clase de talla; $\mathrm{D}=$ 1511,6; E= \% de la producción total de huevos; F= índice de productividad de clase de tamaño (E/A) / Estimation of the index of reproductive potential (IRP) for Panulirus gracilis in Santa Rosa, Manabí. A= number of females per size class/total females, B= proportion of ovigerous females by size class, $C=$ average number of eggs per size class; $D=1511.6 ; E=\%$ total egg production; $F=$ productivity index size class ( $E / A$ )

\begin{tabular}{|c|c|c|c|c|c|c|c|c|c|c|c|}
\hline Clases de talla & $\begin{array}{c}\text { Hembra } \\
\text { totales }\end{array}$ & $\begin{array}{l}\text { hembras } \\
\text { ovígeras }\end{array}$ & A & B & $\mathrm{C}$ & $\begin{array}{c}\text { desoves } \\
\text { por año }\end{array}$ & $\begin{array}{c}\text { Fecundidad } \\
\text { anual } \\
\left(\mathrm{x} 10^{3}\right)\end{array}$ & IPR & $\begin{array}{l}\text { Huevos } \\
\text { por año } \\
\left(\mathrm{x} 10^{3}\right)\end{array}$ & $\mathrm{E}$ & $\mathrm{F}$ \\
\hline $55-60$ & 1 & 0 & 0,37 & - & - & 3 & - & - & - & - & - \\
\hline $60-65$ & 0 & 0 & 0 & - & - & 3 & - & - & - & - & - \\
\hline $65-70$ & 5 & 1 & 1,83 & 20,00 & 123,26 & 3 & 369,77 & 0,90 & 369,77 & 0,28 & 0,16 \\
\hline $70-75$ & 33 & 8 & 12,09 & 24,24 & 233,99 & 4 & 935,94 & 18,14 & $7.487,53$ & 5,75 & 0,48 \\
\hline $75-80$ & 46 & 14 & 16,85 & 30,43 & 273,41 & 4 & $1.093,66$ & 37,10 & $15.311,20$ & 11,77 & 0,70 \\
\hline $80-85$ & 92 & 27 & 33,70 & 29,35 & 382,10 & 4 & $1.528,38$ & 100,00 & $41.266,26$ & 31,71 & 0,94 \\
\hline $85-90$ & 47 & 10 & 17,22 & 21,28 & 400,90 & 4 & $1.603,60$ & 38,86 & $16.035,97$ & 12,32 & 0,72 \\
\hline $90-95$ & 33 & 13 & 12,09 & 39,39 & 503,43 & 4 & $2.013,74$ & 63,44 & $26.178,58$ & 20,12 & 1,66 \\
\hline $95-100$ & 14 & 9 & 5,13 & 64,29 & 652,26 & 4 & $2.609,05$ & 56,90 & $23.481,41$ & 18,04 & 3,52 \\
\hline $100-105$ & 2 & 0 & 0,73 & - & - & 4 & - & - & - & - & - \\
\hline Total & 273 & 82 & 100 & & & & & & & & \\
\hline
\end{tabular}

Los valores obtenidos de IPR por clase de talla muestran que las hembras a partir de $90 \mathrm{~mm}$ LC presenta aproximadamente el doble de potencial reproductivo que las hembras menores a $90 \mathrm{~mm} \mathrm{LC}$, lo cual podría indicar que desde esta talla las hembras pueden ser consideradas mega reproductores. Consecuentemente, las hembras menores a $80 \mathrm{~mm}$ LC aportaron con el 17,8\% de la producción total de huevos, mientras que las hembras entre 90-100 mm LC aportaron con el 38,2\% lo que coincide con el mayor índice de productividad encontrado por clase de tallas con 5,2 (Tabla 1).

\section{Discusión}

Los resultados muestran una talla media de $83 \mathrm{~mm} \mathrm{LC} \pm$ 8,8, los cuales coinciden con los resultados encontrados por Pérez (2011) en México y Figueroa \& Mero (2013) en Ecuador, con $76 \mathrm{~mm}$ LC y $73 \mathrm{~mm}$ LC, respectivamente. Sin embargo, organismos de menor talla (61 mm LC) fueron reportados para Jaramijó, Ecuador por Delhaye \& Ormaza (2006).

Estas diferencias en la talla media de captura podrían deberse a que en la zona de estudio (Santa Rosa) la presión pesquera es menor a la de Puerto Cayo (Figueroa \& Mero 2013) y Jaramijó (Delhaye \& Ormaza 2006). Santa Rosa presenta cambios repentinos en sus condiciones oceanográficas, pasando de suaves corrientes a corrientes de resaca, fuertes vientos, oleaje alto y neblina, lo cual complica las operaciones de pesca y crea una barrera natural que hace poco accesible la zona para los pescadores.

La estructura de tallas en las poblaciones de langostas espinosas puede ser afectada por el arte de pesca utilizado tal como lo mencionan Figueroa \& Mero (2013) quienes encontraron diferencias significativas en la talla media de captura por método de pesca, donde las langostas capturadas con redes tienden a ser más pequeñas en comparación con las que son capturadas con hookah. Otro factor importante que puede afectar a estructura de tallas es la intensidad de la presión pesquera o por patrones de movimiento de los organismos a lo largo de la costa (Lyons et al. 1981, Padilla \& Briones 1997), lo cual ocasiona que en zonas de alta presión pesquera exista una mayor frecuencia de organismos pequeños. Esta hipótesis es reafirmada por Cox \& Hunt (2005), quienes encontraron mayor frecuencia de langostas grandes en áreas marinas protegidas donde la explotación pesquera es mínima en comparación con áreas marinas sobre explotadas.

En el presente estudio se encontró que el 30, 5 y $6 \%$ del total de hembras capturadas corresponden al estadio 
reproductivo III, IV, y V, respectivamente. La alta frecuencia de hembras en estadio III puede estar en razón de varios factores entre los cuales se encuentra el tiempo de incubación de huevos, tal como lo indican Senthil et al. (2005) para Panulirus ornatus (Fabricius, 1798), donde estimaron que el tiempo total de incubación de los huevos en este estadio es de 22 a 26 días, mientras que Sachlikidis et al. (2010) demuestran para la misma especie que el tiempo de incubación de los huevos está relacionado con la temperatura del agua, de tal manera que si se aumenta la temperatura disminuye el tiempo de incubación. En concordancia con esto, Arzola et al. (2007) encontraron para Panulirus inflatus (Bouvier, 1895) y P. gracilis resultados similares a los presentes, con una alta frecuencia de estadio III, pero lo atribuyen a que los huevos permanecen en esta etapa de desarrollo por más tiempo en comparación con el estadio IV y V. Por su parte Lozano et al. (1991) y Bertelsen (2013), exponen en sus resultados que Panulirus argus (Latreille, 1804) realiza migraciones hacia zonas más profundas $(>15 \mathrm{~m})$ para la incubación de huevos, razón por la cual las hembras en estadios IV y V no se encuentran en las zonas someras. Waddington et al. (2005) indican que las hembras en su primer estadio de incubación de huevos (estadio III) tienen mayor actividad locomotora que las hembras que han desovado recientemente, lo cual las hacen más propensas a ser capturadas en el momento que se encuentran en busca de alimento. Por lo expuesto, la alta abundancia de hembras ovígeras en estadio reproductivo III en Santa Rosa se debe posiblemente a la profundidad en la que fueron capturadas (7-12 m), el tiempo que toma el desarrollo de la masa de huevos en estadio III y eventualmente a la afinidad de las hembras ovígeras a sustratos mixtos (característicos de bajos rocosos) en busca de alimento que supla sus requerimientos nutricionales en estas etapas reproductivas (McKoy \& Leachman 1982, Castillo et al. 2013).

En el presente estudio, el análisis de regresión entre la fecundidad y LC indican que estas variables se encuentran correlacionas en un $82 \%$ y el modelo explica el $67 \%$ de esta relación, coincidiendo con lo reportado por Tapia \& Castro (2000) para Panulirus interruptus (Randall, 1840) quienes afirman una correlación del 84,6\% con un coeficiente de determinación de 71,6\%. Estos coeficientes de determinación relativamente bajos para la fecundidad pueden estar en razón de la variabilidad de factores abióticos que influyen en los procesos reproductivos tales como la temperatura, el fotoperiodo y el gradiente latitudinal (Waddy \& Aiken 1992), por tales motivos es necesario considerar estos factores en el diseño de muestreo para futuros estudios de fecundidad de langostas espinosas.

Se determinó que una hembra de $P$. gracilis en estadio reproductivo III puede alojar en su abdomen un promedio de $382.095 \pm 74.026$ huevos en el rango de talla comprendido entre 80 a $85 \mathrm{~mm}$ LC. Resultados similares encontraron Velázquez et al. (2010) con la fecundidad promedio de 384.314 para $P$. inflatus, al igual que Vijayakumaran et al. (2012) que determinaron en el mismo rango de talla un número de 332.617 y 351.592 huevos para Panulirus homarus (Linnaeus, 1758) y Panulirus versicolor (Latreille, 1804), respectivamente. Estos resultados difieren con lo reportado por Domínguez (2003), quien describe para $P$. gracilis en el Pacífico mexicano una fecundidad superior con 598.607 (1992) y 618.342 (1995) huevos. Por su parte, otros autores registran una fecundidad inferior a $P$. gracilis (presente estudio) tales como Fonseca \& Briones (1998) con 289.000 huevos para P. argus y Tapia \& Castro (2000) quienes encontraron un promedio de 311.714 huevos para $P$. interruptus.

La variación en la producción de huevos se ve influenciada tanto a nivel intraespecífica (debido a la plasticidad en el crecimiento y la fecundidad), así como a nivel interespecífico (características determinadas genéticamente de reproducción, el tamaño del huevo, etc.), y consecuentemente la producción de huevos de una hembra durante todo su ciclo de vida generalmente es proporcional a las tasas de mortalidad a las que se encuentra sometida la especie hasta llegar a la etapa adulta (Pollock 1997).

Es evidente que las langostas del género Panulirus poseen una alta variabilidad en la producción de huevos por cada desove, esto puede explicarse por las preferencias de hábitats de cada especie y por lo tanto por características biológicas y ecológicas distintas. Inclusive una misma especie puede presentar diferencias en la fecundidad, tal como lo indica Pineda et al. (1981) quienes encontraron para $P$. interruptus diferencias en el número de huevos producidos por cada hembra de acuerdo con el gradiente latitudinal, es decir, organismos que se encuentran distribuidos en zonas cercanas a la línea ecuatorial presentan mayor fecundidad que los organismos de la misma especie que se encuentran más alejados de la zona ecuatorial.

Otro factor que puede afectar la producción de huevos es el aumento de la variabilidad de las condiciones medioambientales y el aumento de las tasas de 
depredación (Pollock 1995). En relación a esta hipótesis, Senthil et al. (2005) reportan para $P$. ornatus una fecundidad de 541.894 huevos obtenida en cautiverio, siendo mucho más baja que la media de 1.121.507 determinada en medio natural para la misma especie. Esto sugiere influencia de factores medio ambientales que no pueden ser reproducidos en cautiverio.

Según Kanciruk \& Herrnkind (1976), el IPR permite determinar la clase de talla de las hembras ovígeras que contribuyen con la mayor cantidad de huevos a la población. En concordancia con esto, Bohnsack et al. (2000) mencionan que las poblaciones pesqueras deben tener un potencial reproductivo del $50 \%$ con un mínimo de $30 \%$ para mantenerse estables y sin riesgo de colapso. Por ende, si el potencial reproductivo es menor al $30 \%$ no habría el aporte suficiente de nuevos individuos a las poblaciones, ocasionando el inicio de un proceso de sobreexplotación del recurso.

Los resultados muestran que las hembras con tallas entre 80-100 mm LC aportan con el 82\% de la producción total de huevos y representan el $68 \%$ de la población muestreada en Santa Rosa, lo cual revela claramente que se está explotando la porción de la población que sirve de soporte a toda la pesquería. Lyons et al. (1981) al respecto sostienen que la producción de huevos de $P$. argus en Florida Keys disminuyó en un $88 \%$ debido a la eliminación de las hembras grandes de la población, mientras que Pérez et al. (2002) encontraron que la captura de langostas que están por debajo de la talla legal ocasiona un desequilibrio en los procesos biológicos del stock tales como cambios en la talla de primera madurez sexual y la fecundidad media de la población. Beverton (1992) sostiene que una población con una pesquería sustentable debe tener la talla de captura sobre la talla de primera madurez sexual. Por su parte, Myers et al. (1999) menciona que las poblaciones cuyos individuos logran desovar por lo menos una vez antes de ser capturados tendrán una pesquería sustentable y la población no colapsará.

Por otra parte, los especímenes con tallas menores a $80 \mathrm{~mm}$ LC contribuyen con el 17,8\% de la producción de huevos y constituyen el $31 \%$ del total de la captura. De acuerdo con los resultados de Castillo et al. (2013) provenientes de las capturas comerciales, estos especímenes se encuentran por debajo de la talla media de madurez sexual ( $83 \mathrm{~mm} \mathrm{LC}$ ), y por lo tanto esta parte de la población aún se encuentra prioritariamente en el proceso de crecimiento y no de constante reproducción.
Los resultados que se obtienen utilizando la ecuación propuesta por Kanciruk \& Herrnkind (1976), pueden subestimar la producción real de huevos de una población de langostas, debido a que esta ecuación es dependiente del número de especímenes de hembras ovígeras por clase de talla influyendo en los resultados obtenidos. Por consiguiente, dependiendo de la naturaleza de los datos y del tipo de muestreo (buceo, redes, trampas, profundidades), la frecuencia de tallas y el número hembras ovígeras puede variar considerablemente, tal como lo demuestra Figueroa \& Mero (2013), quienes determinan diferencias en la estructura de tallas y frecuencia de hembras ovígeras y no ovígeras de acuerdo al arte de captura utilizado, por ende ocasionando que el potencial reproductivo de una población pueda ser sub o sobre estimado. Por tales motivos es importante organizar una estructura de muestreo que permita obtener información representativa de la población para futuros estudios.

Factores adicionales que pueden influir en el potencial reproductivo son los sugeridos por Montgomery (1995) y Padilla \& Briones (1997) quienes sostienen que la localidad de pesca y/o patrón de movimiento de las langostas a lo largo de la costa podrían influir en la composición de tallas de la captura. En el presente estudio los datos se obtuvieron por medio del monitoreo de desembarques pesqueros de un arte de pesca no selectivo (captura con redes), por lo tanto, los resultados de potencial reproductivo son cercanos a la realidad del recurso, ya que los datos obtenidos no se encuentran sesgados a un rango de tallas específico.

Finalmente, Yi et al. (2007) concluyen que el análisis del potencial reproductivo nos proporciona información crítica, necesaria para el establecimiento de una regulación de tamaño máximo de captura. Sin embargo, una mejor comprensión de la dinámica de poblaciones requiere también el entendimiento de los procesos claves del ciclo de vida, tales como crecimiento, mortalidad, y el mecanismo de dispersión de las larvas (Csirke 1980, Negrete et al. 2002).

Tomando como base que la talla media de madurez sexual de $P$. gracilis se estimó en $83 \mathrm{~mm} \mathrm{LC} \mathrm{(Castillo} \mathrm{et} \mathrm{al.}$ 2013) y considerando que la talla óptima de captura debe ser mayor a la talla media de madurez sexual (Froese 2004), podemos sugerir de acuerdo a nuestros resultados la imposición de una talla máxima de captura de los especímenes mayores a $90 \mathrm{~mm} \mathrm{LC} \mathrm{(228} \mathrm{mm} \mathrm{LT)} \mathrm{ya} \mathrm{que}$ estos pueden aportar con el $38 \%$ de la producción total 
de huevos, adoptando la estrategia de proteger a los mega reproductores propuesta por Froese (2004), contribuyendo de esta manera a la sostenibilidad de la pesquería de la langosta en Santa Rosa, Manabí, Ecuador. Sin embargo, es necesario complementar los resultados del presente estudio de potencial reproductivo en $P$. gracilis con estudios de histología gonadal en toda la costa continental de Ecuador.

\section{Agradecimientos}

A Cristiano De Matos, Georgina Gluyas y Dayanara Macías Mayorga por la revisión y sugerencias hechas al manuscrito. A la Unidad de Sistemas de Información Geográfica (UNISIG-ULEAM). A los estudiantes pasantes y auxiliares de investigación del Programa Langosta por su colaboración. Este estudio fue financiado por la Universidad Laica Eloy Alfaro de Manabí.

\section{LiTERATURA CITADA}

Arzola J, L Flores, M Ortiz \& Y Gutiérrez. 2007. Captura y aspectos reproductivos de la pesquería de las langostas Panulirus inflatus y Panulirus gracilis (Crustacea: Decapoda) en el sur de Sinaloa, México. Ciencia y Mar 11(31): 15-22.

Bertelsen D. 2013. Characterizing daily movements, nomadic movements, and reproductive migrations of Panulirus argus around the Western Sambo Ecological Reserve (Florida, USA) using acoustic telemetry. Fisheries Research 144: 91-102.

Beverton R. 1992. Patterns of reproductive strategy parameters in some marine teleost fishes. Journal of Fish Biology 41: $137-160$.

Bohnsack J, B Causey, M Crosby, R Griffis, M Hixon, T Hourigan, K Koltes, J Maragoss, A Simons \& J Tilmant. 2000. A rationale for minimum $20-30 \%$ no-take protection. In: Proceedings 9th International Coral Reef Symposium, Bali, pp. 23-27.

Briones P \& E Lozano. 2003. Factors affecting growth of the spiny lobsters Panulirus gracilis and Panulirus inflatus (Decapoda: Palinuridae) in Guerrero, México. Revista de Biología Tropical 51: 165-174.

Briones P, E Lozano, A Martínez \& A Cortés. 1981. Aspectos generales de la biología y pesca de la langosta en Zihuatanejo, Guerrero, México. Anales del Instituto de Ciencias del mar y Limnología 8(1): 1-314.

Cadima E. 2003. Manual de evaluación de recursos pesqueros. FAO Documento Técnico de Pesca 393: 1-162.

Castillo R, J Figueroa, D Mero \& C Erazo. 2013. Langosta verde Panulirus gracilis. Avances en estudios biológicos y ecológicos para la construcción de estrategias de conservación y manejo. Hippocampus Revista Científica, Colección Recursos Marinos 2: 1-47.
Castrejón M. 2012. Evaluación de la cadena de valor de la pesquería de la langosta espinosa (Panulirus penicillatus y P. gracilis) en la reserva marina de Galápagos. En: Ramírez J, M Castrejón \& MV Toral-Granda (eds). Mejorando la pesquería de la langosta espinosa en la Reserva Marina de Galápagos, pp. 150-156. WWF Galápagos, Quito.

Correa J, M Herrera, G Villanueva \& J Campos. 1994. Informe sobre el recurso langosta en la región insular y continental del Ecuador, 7 pp. Instituto Nacional de Pesca. Departamento de Ecología Marina, Quito.

Cox C \& J Hunt. 2005. Change in size and abundance of Caribbean spiny lobsters Panulirus argus in a marine reserve in the Florida Keys National Marine Sanctuary, USA. Marine Ecology Progress Series 294: 227-239.

Csirke J. 1980. Introducción a la dinámica de poblaciones de peces. FAO Documento Técnico de Pesca 192: 1-82.

Cun M \& J Campos. 1993. Estudio de las capturas comerciales de Panulirus gracilis (Langosta verde) del noreste del golfo de Guayaquil (1970-1979). Boletín Científico Técnico Instituto Nacional de Pesca Ecuador 12: 1-19.

Delhaye L \& C Ormaza. 2006. Aspectos biométricos de las capturas de langosta espinosa Panulirus gracilis (Streets, 1871) en las costas de Jaramijó, Manabí, Ecuador. Tesis de Licenciatura, Facultad Ciencias del Mar, Universidad Laica Eloy Alfaro de Manabí, Manta, 76 pp.

De Martini E, M Ellis \& V Honda. 1993. Comparisons of spiny lobsters Panulirus marginatus fecundity, egg, size, and spawning frequency before and after exploitation. Fishery Bulletin 91: 1-7.

De Martini E, G Di Nardo \& H William. 2003. Temporal changes in population density, fecundity, and egg size of the Hawaiian spiny lobsters (Panulirus marginatus) at Necker Bank Northwestern Hawaiian Island. Fishery Bulletin 101: 22-31.

Domínguez J. 2003. Fecundidad de la langosta espinosa Panulirus inflatus (Bouvier) en el litoral de Nayarit. Tesis de Licenciatura, Facultad Ciencias del Mar, Universidad Autónoma de Sinaloa, Sinaloa, 68 pp.

Figueroa J \& D Mero. 2013. Talla de captura y reproducción de la langosta verde Panulirus gracilis (Decapoda: Palinuridae) en la costa de Manabí, Ecuador. Revista de Biología Tropical 61(3): 1189-1199.

Figueroa J, R Castillo, D Mero \& C Erazo. 2013. Langosta verde Panulirus gracilis. Un recurso que debemos investigar para preservar. Hippocampus Revista Científica, Colección Recursos Marinos 1: 1-27.

Fonseca M \& P Briones. 1998. Fecundity of the spiny lobster Panulirus argus (latreille, 1804) in the caribbean coast of Mexico. Bulletin of Marine Science 63(1): 21-32.

Froese R. 2004. Keep it simple: three indicators to deal with overshing. Fish and Fisheries 5: 86-91. 
García H. 2004. Índice de potencial reproductivo de la langosta Panulirus gracilis (Decapoda: Palinuridae) en la costa centro-sur de Nayarit. Tesis de Licenciatura, Universidad Autónoma de Sinaloa, Sinaloa, 45 pp.

Gracia A. 1985. Variación estacional en la fecundidad de la langosta Panulirus inflatus (Bouvier 1895) (Crustacea: Decapoda: Palinuridae). Ciencias Marinas 11: 7-27.

Green B, C Gardner \& R Kennedy. 2009. Generalised linear modelling of fecundity at length in southern rock lobsters, Jasus edwardsii. Marine Biology 156: 1941-1947.

Guzmán H, R Cipriani, A Vega, M López \& J Meir. 2008. Population assessment of the pacific green spiny lobster (Panulirus gracilis) in the pacific of Panama. Journal of Shellfish Research 43: 14-23.

Kanciruk P \& F Herrnkind. 1976. Autumnal reproduction in Panulirus argus at Bimini, Bahamas. Bulletin of Marine Science 26(4): 417-432.

Lozano E, P Briones \& B Phillips. 1991. Fishery characteristics, growth, and movements of the spiny lobster Panulirus argus in Bahía de la Ascension, Mexico. Fishery Bulletin 89: 79-89.

Lyons W, D Barber, S Foster, F Kennedy \& G Milano. 1981. The spiny lobster, Panulirus argus, in the middle and upper Florida Keys: population structure, seasonal dynamics, and reproduction. Florida Marine Research Publications 38: 1-38.

McKoy J \& A Leachman. 1982. Aggregations of ovigerous female rock lobsters, Jasus edwardsii (Decapoda: Palinuridae). New Zealand Journal of Marine and Freshwater Research 16(2): 141-146.

Montgomery S. 1995. Patterns in landings and size composition of Jasus verreauxi (H. Milne Edwards, 1851) (Decapoda, Palinuridae), in waters off New South Wales, Australia. Crustaceana 68(2): 257-266.

Myers R, K Bowen \& N Barrowman. 1999. Maximum reproductive rate of fish at low population sizes. Canadian Journal of Fisheries and Aquatic Sciences 56: 2404-2419.

Naranjo H. 2012. Patrón reproductivo y talla media de madurez sexual de hembras de la langosta Panulirus gracilis (Decapoda: Palinuridae) en Playa Lagarto, Guanacaste, Costa Rica. Revista de Biología Tropical 60(4): 1783-1793.

Negrete F, E Lozano \& P Briones. 2002. Population dynamics of the spiny lobster Panulirus gattatus (latreille) in a coral reef on the Mexican Caribbean. Journal of Shellfish Research 21(1): 279-288

Padilla S \& P Briones. 1997. Biological characteristics of the spiny lobsters (Panulirus spp.) from the commercial catch in Puerto Morelos, Quintana Roo, Mexico. Ciencias Marinas 23(2): 175-193.

Pérez R. 2011. Catch composition of the spiny lobster Panulirus gracilis (Decapoda: Palinuridae) off the western coast of Mexico. Latin American Journal of Aquatic Research 39(2): 225-235.
Pérez R, I Muñoz, L Valadez \& M Borrego. 2002. The current status of the fishery for spiny lobsters Panulirus inflatus and $P$. gracilis (Decapoda: Palinuridae) along the Mexican Pacific coast. In: Hendrickx ME (ed). Contributions to the study of east Pacific crustaceans, Vol. 1: 327-347. Instituto de Ciencias del Mar y Limnología, Universidad Nacional Autónoma de México, México.

Pineda J, A Díaz de León \& F Uribe. 1981. Fecundidad de la langosta roja Panulirus interruptus (Randall, 1940) en Baja California. Ciencia Pesquera, Instituto Nacional de Pesca, México 1(1): 99-118.

Pollock D. 1995. The evolution of life history patterns in three genera of spiny lobsters. Bulletin of Marine Science 57: 516-526.

Pollock D. 1997. Egg production and life-history strategies in some clawed and spiny lobster populations. Bulletin of Marine Science 61: 97-109.

Ramírez J, M Castrejón \& M Toral. 2012. Mejorando la pesquería de la langosta espinosa de la Reserva Marina de Galápagos, 267 pp. WWF Galápagos, Quito.

Sachlikidis N, C Jones \& J Seymour. 2010. The effect of temperature on the incubation of eggs of the tropical rock lobster Panulirus ornatus. Aquaculture 305: 79-83.

Seijo J, O Defeo \& S Salas. 1997. Bioeconomía pesquera. Teoría, modelación y manejo. FAO Documento Técnico de Pesca 368: 1-176.

Senthil T, M Remany, T Leema, J Kumar, J Santhanakumar, M Vijayakumaran, R Venkatesan \& M Ravindran. 2005. Growth, repetitive breeding, and aquaculture potential of the spiny lobster, Panulirus ornatus. New Zealand Journal of Marine and Freshwater Research 39(2): 311-315.

Tapia O \& J Castro. 2000. Fecundidad y anatomía microscópica del ovario de la langosta roja Panulirus interruptus de Punta Eugenia, BCS., México. Ciencia Pesquera 14: 63-66.

Velázquez J, M Villalejo \& A Tripp. 2010. Fecundidad y proporción de sexos de Panulirus inflatus en la costa occidental del Golfo de California, México. Revista de Biología Marina y Oceanografía 45(1): 71-76.

Vijayakumaran M, A Maharajan, S Rajalakshmi, P Jayagopal, M Subramanian \& M Remani. 2012. Fecundity and viability of eggs in wild breeders of spiny lobsters, Panulirus homarus (Linnaeus, 1758), Panulirus versicolor (Latrielle, 1804) and Panulirus ornatus (Fabricius, 1798). Journal of the Marine Biological Association of India 54(2): 18-22.

Villón C, I Cedeño, J Correa \& M Peralta. 2000. Situación actual del recurso langosta (Panulirus gracilis) en la costa continental ecuatoriana: Opciones de manejo para la pesquería, 12 pp. Instituto Nacional de Pesca, Quito. 
Waddington K, R Melville, D Walker \& B Knott. 2005. Effect of reproductive state and sex on movement and food consumption of western rock lobster (Panulirus cygnus) in a tank environment. New Zealand Journal of Marine and Freshwater Research 39(2): 365-372.

Waddy S \& D Aiken. 1992. Seasonal variation in spawning by preovigerous American lobster, Homarus americanus, in response to temperature and photoperiod condition. Canadian Journal of Fisheries and Aquatic Sciences 49: 1114-1117.
Yi C, S Chi, Y Chen, Y Su \& C Wei. 2007. Reproductive biology of the spiny lobster, Panulirus Penicillatus, in the southeastern coastal waters off Taiwan. Marine Biology 151: 553-564.

Recibido el 2 de julio 2014 y aceptado el 2 de enero de 2015

Editor: Claudia Bustos D. 